Acta Technologica Agriculturae 3

Nitra, Slovaca Universitas Agriculturae Nitriae, 2020, pp. 150-154

\title{
USING ACOUSTIC EMISSION FOR MEASURING SURFACE ROUGHNESS
}

\author{
Jakub ROZLIVKA ${ }^{1}$, Václav KAŠPAR ${ }^{1}$, Petr DOSTÁL ${ }^{1}$, Michal ČERNÝ1, \\ Benjamín HAJTMAN ${ }^{1}$, Jozef ŽARNOVSKÝ2* \\ ${ }^{1}$ Mendel University in Brno, Czech Republic \\ ${ }^{2}$ Slovak University of Agriculture in Nitra, Slovakia
}

\begin{abstract}
This paper is focused on exploring and utilizing the acoustic emission and its behaviour during surface roughness measurement. Surface quality or coating properties significantly affect the reliability and durability of operations. Three samples were selected for an experiment to demonstrate the possibility of measuring the roughness of surface textures by means of acoustic emission method (AE). These samples were made of the following materials: sample A2 - EN 54SiCr6 steel formed in water, austenitized at $850^{\circ} \mathrm{C}$ for 20 minutes, sample A3 - non-heat-treated spheroidal graphite cast iron, and sample B5 - abrasion resistant austenitic manganese steel. The surfaces were subjected to the same surface treatments (roughness $R a=1.6-3.2 \mu \mathrm{m}$ ) and measured under the same conditions. All possible measurements were measured on both $x$ - and $y$-axes. Final results are presented graphically. The measured $A E$ values showed a visible effect in the AE signals due to the lack of surface roughness.
\end{abstract}

Keywords: acoustic emission; surface roughness; surface quality; surface texture; acoustic waves

Evaluation of the surface properties of the parts is a result of a specific technological process depending on specific operational conditions of the surface in operation. This surface rating is frequently referred to as surface integrity. According to Dias et al. (2016) and Barényi et al. (2019), the concept of surface integrity can be defined as all properties of a functional surface, which have direct impact on its performance under operation and result from technological methods used for its manufacturing; these are also reflected in the quality of the machined surface manufactured components. The most frequently evaluated surface integrity parameters include microgeometry (surface structure); surface hardening layer after machining; and structural changes in coating (Junki et al., 2018; Gong et al., 2018; Dobrocký and Kusmič, 2015; Barényi et al., 2019).

Surface represents a border separating objects or substances from each other. One can subdivide the surface into three groups (nominal, real and measured) (Bhushan, 2001).

Nominal surface (ideal surface) represents the intended surface without any surface irregularities. Its profile and dimensions are usually shown in a drawing. The nominal or ideal surface structure does not consider the intended surface roughness (Bhushan, 2001).

Real surface is the real shape of an object and actual boundary of an object. It differs from the nominal surface due to the surface-shaping processes taking place. Differences also come from the properties, composition and structure of the body material (Bhushan, 2001).

Measured surface is shown using a measuring device that obtains values by measuring the actual surface. The real and measured surfaces differ, because no measuring method is capable of perfect interpretation of the actual surface (Bhushan, 2001).

Surface parameters are repeated or irregular deviations from the ideal surface and form a surface texture. Surface texture includes (a) roughness (nano-roughness and microroughness); (b) waviness (macro-roughness); (c) lay; and (d) flaws.

Nano-roughness and micro-roughness are shaped by fluctuations of short wavelengths on the surface, indicated by ridges (asperities) (local maxima) and valleys (local minima) of changeable amplitudes and distances. Their size can be described as large in contrast to the molecular dimensions (Bhushan, 2013). Within the roughness sampling length, nano-roughness and micro-roughness are considered to include traverse feed marks and other errors (Votava et al., 2020; Ohtsu, 1995; Miller et al., 2005). Amplitude parameters are considered to be the most important indicators for surface texture assessment. Measurements of vertical surface deviations are described herein (Machek, 2013).

Waviness is a surface irregularity formed by surface layers of longer wavelength; it can be a result of device deflection, vibration, heat treatment, and uneven pressure of the work tool on the material. Irregularities are associated with waviness, the length of which exceeds the roughness sampling length but does not exceed the waviness sampling length (Votava et al., 2020; Ohtsu,1995, Miller et al., 2005).

Lay is determined by the main direction of the predominant surface pattern, which is usually defined by the manufacturing process (Poláková and Dostál, 2019; Ohtsu, 1995)

Contact address: Jozef Žarnovský, Slovak University of Agriculture in Nitra, Department of Quality and Engineering Technologies, Tr. Andreja Hlinku 2, 94976 Nitra, Slovak Republic, e-mail: jozef.zarnovsky@uniag.sk 
Flaws are unintentional, unexpected and undesirable surface texture errors. Furthermore, the surface may contain significant deviations from the nominal shape due to a very long wavelength, resulting in shape errors. They are generally not considered to be part of the surface (Ohtsu, 1995).

Acoustic emission (AE) is a promising and challenging subject of modern technology and science. It is defined as the generation of sound and ultrasound waves in materials subjected to deformation forces. At the time of fracture, cracking occurs with the release of stored stress energy. Due to microcracking, some of the stored energy is released in the form of elastic waves - acoustic emissions. As illustrated in Fig. 7, AE waves spread through an object and can be recorded by an $A E$ sensor on the surface, which transforms the vibrations into electric signals (Ohtsu, 1995, Miller et al., 2005).

The propagation of fracture sound in materials was originally marked as $A E$ because they are both acoustic and audible. It has been explained, on the basis of the elastodynamics, that $A E$ waves may be synthesized as elastic waves due to dislocation movement. The latter part of the $A E$ waveform is generally the result of the $A E$ sensor resonance vibration (Junki et al., 2018; Gong et al., 2018; Dobrocký et al., 2019).

The volume and characteristics of generated $A E$ waves are based on the source characteristics: initial significance; current state; local metallurgical structure; and current environments. There is a couple of factors which effect the propagation of the wave in the material. Surface waves are created by reflections that are caused by macro-discontinuities and micro-discontinuities. Grain boundaries, inclusion, etc. lead to reflection and diffraction. The anisotropic properties of the medium cause the wave to change its speed and disperse in different directions with different speeds, showing the non-ideal elastic behaviour of the medium (Ohtsu, 1995; Miller et al., 2005).

The paper aims to show the diversity of acoustic emission utilization and possibilities of its use in practice. A new method of surface roughness measurement using acoustic emission is proposed and AE surface scanning procedure was designed. This procedure was subsequently tested using the surfaces of various types of materials.

\section{Material and methods}

Three samples made of different materials were selected to demonstrate the ability to measure surface texture roughness using the designed method. The surfaces were subjected to the same surface treatment (roughness $R a=$ 1.6-3.2 $\mu \mathrm{m}$ ) and measured under the identical conditions (Tulka, 2005). Each sample was marked with a combination of letter and number for simplification:

- A2 - Steel EN 54SiCr6 hardened in water, austenitized at $850^{\circ} \mathrm{C}$ for 20 minutes (Fig. 1);

- A3 - Spheroidal graphite cast iron without heat treatment; - B5 - Abrasion resistant austenitic manganese steel (EN X53CrMnNiN21 9), especially resistant to dynamic abrasive wear (so-called 13\% Mn "HADFIELD steel").

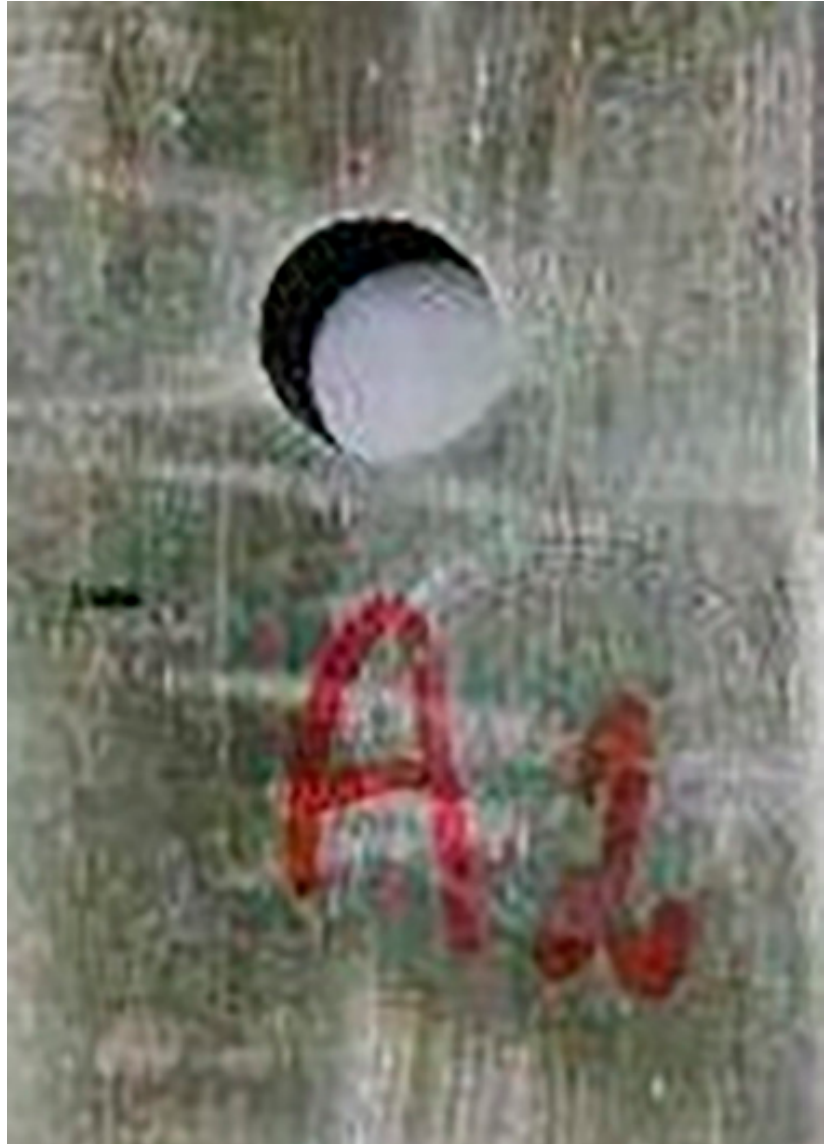

Fig. 1 Measured samples

An AE Xedo analyser manufactured by Dakel was used for the purposes of measuring the acoustic signal. Amount of released energy during the measurements was recorded utilizing an IDK 09 sensor produced by Dakel; this sensor transferred the released energy to the acoustic signal. Recorded signal was then sent to Xedo analyser and subsequently to PC with DaeShow software, by means of which the measurement plots were created.

Diamond tip was used to produce acoustic signals. Gauge for the tip was tailored specifically to order and was made of stainless steel. A diamond tip with a diameter of approx. $0.75 \mu \mathrm{m}$, with an angle of $60^{\circ}$, was embedded to a stainless steel cylinder and fastened with silver solder (Fig. 2)

For accurate measuring, optimal conditions were provided by isolating the ambient conditions from ambient noise in order not to distort the measurement results. An IDK09 sensor was attached to each sample using a plastic clamp (a special binding medium was added between the meter and the sensor). Several measurements were performed before the measurement. Pen tests were conducted to tune ambient noise, which would distort the entire measurement. DaeShow software was set up to provide the clearest results with almost no noise, following the Pen test.

The distance was measured simultaneously in the $x$-axis and $y$-axis in the sample. The sample was supported with an anti-vibration pad to prevent the propagation of vibrations from the surroundings. 


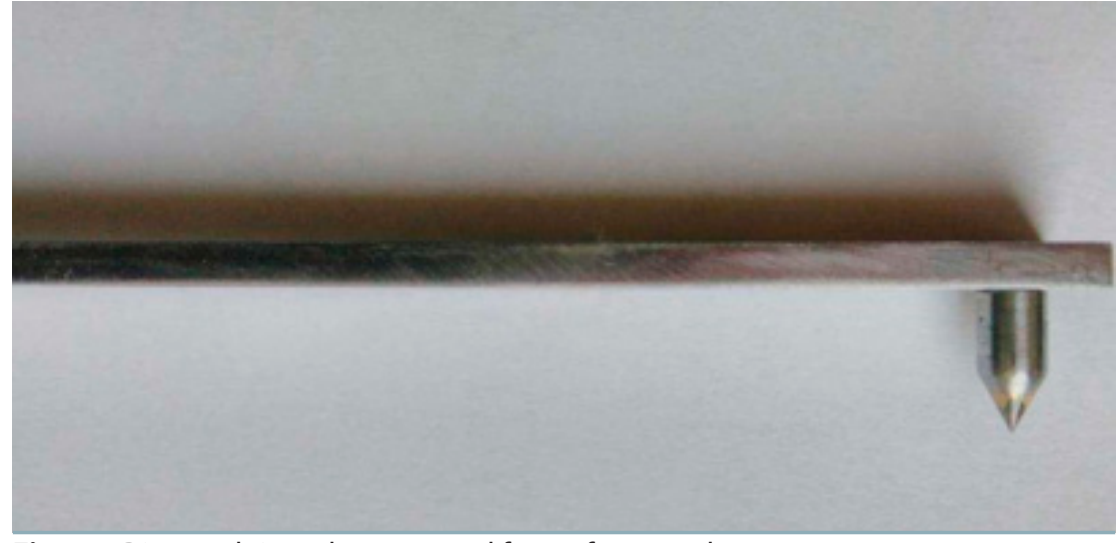

Fig. 2 Diamond tipped gauge used for surface roughness measurement

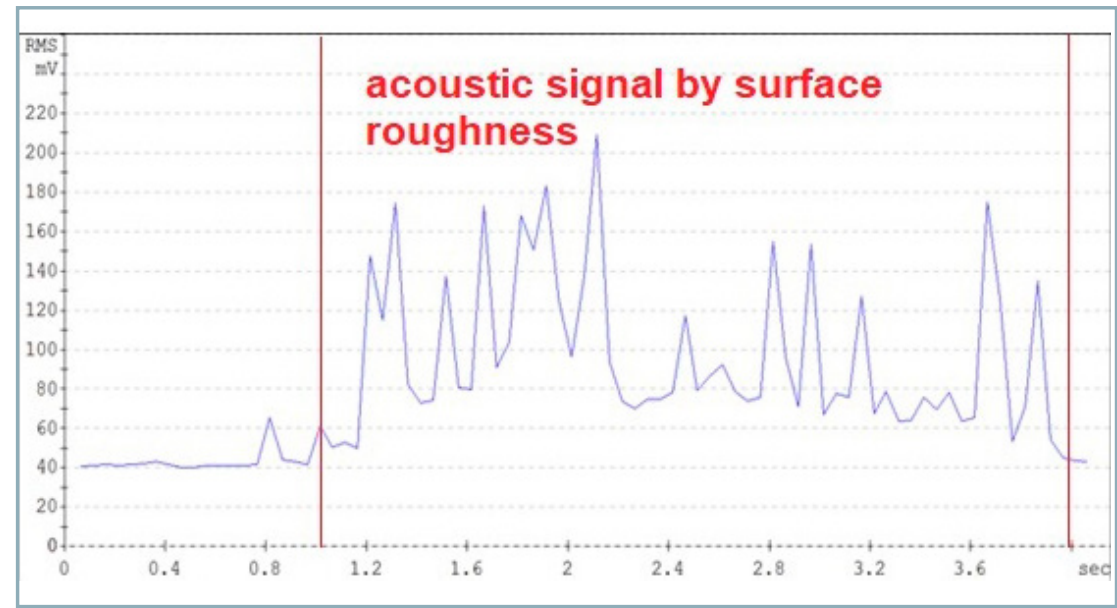

Fig. 3 Sample A2 measured using IDK-09 sensor on the $x$-axis

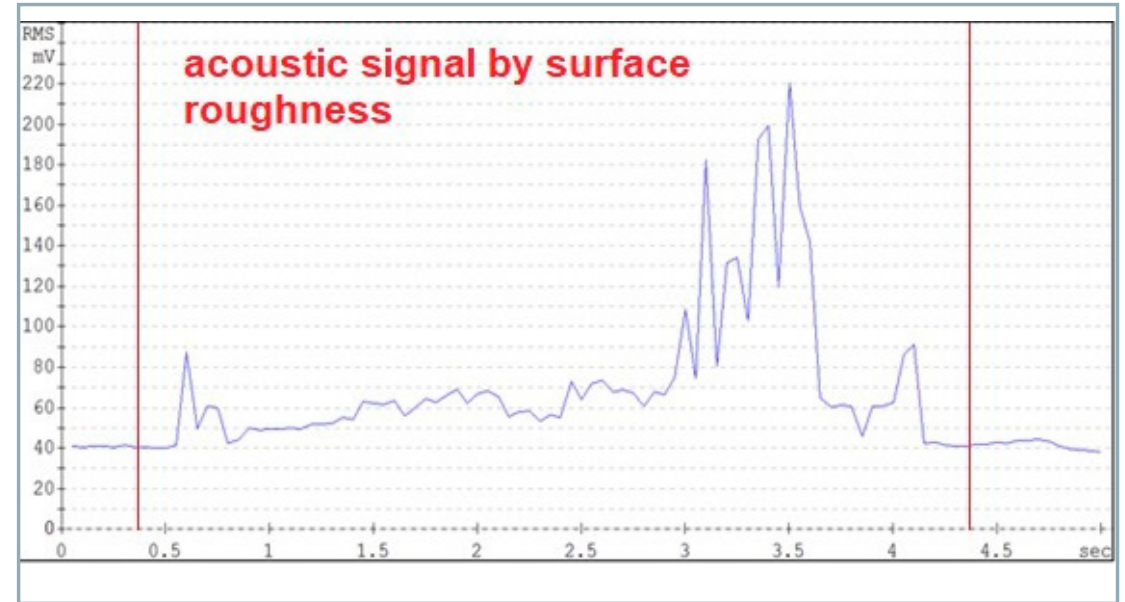

Fig. 4 Sample A2 measured using IDK-09 sensor on the $y$-axis
The measurement record was started when the gauge diamond tip was placed on the sample surface and pulled along the indicated path. The track length was $50 \mathrm{~mm}$. Recording stopped at the end of the track. The measurement was repeated five times and then continued on the $y$-axis.

\section{Results and discussion}

The acoustic surface response of selected materials was measured. For this purpose, a special tool equipped with a diamond tip and a piezoelectric sensor connected to the evaluation device was produced. Samples of three types of material were tested, all with surface roughness in the range $\mathrm{Ra}$ 1.6-3.2 $\mu \mathrm{m}$. The following materials were investigated: steel EN 54SiCr6; spheroidal graphite cast iron without heat treatment; and steel EN X53CrMnNiN21 9, resistant to abrasion. Diamond specimen cross-sections were measured in samples in both the $x$-axis and $y$-axis.

Following graphs show data on the observed sample surfaces obtained by means of IDK-09 sensor fixed on the gauge. On the basis of the obtained results, it is clear that the values measured by AE method differ significantly in various materials.

In relation to testing of EN 54SiCr6 hard steel, a large difference in acoustic response was observed between the $x$ and $y$ measurements. This was due to the directionality of the roughness of the surface created by the defending tool. The recording was performed in the direction and perpendicular direction of the tool track. In the $x$-axis direction, one can see a regular mark of the instrument. On the $y$-axis, the track is irregular, which is caused by the gauge moving along the track (Figs. 3 and 4).

The surface analysis of a spheroidal graphite cast iron material shows a similar signal in the $A E$ record in both measured directions. However, its course was influenced by the graphite contained in the alloy and the presence of graphite in the material affected the recording. After the measurement, there remained a visible trace in the material (Figs. 5 and 6). Moreover, record of the cast iron was distorted despite using less sensitive sensor. The 


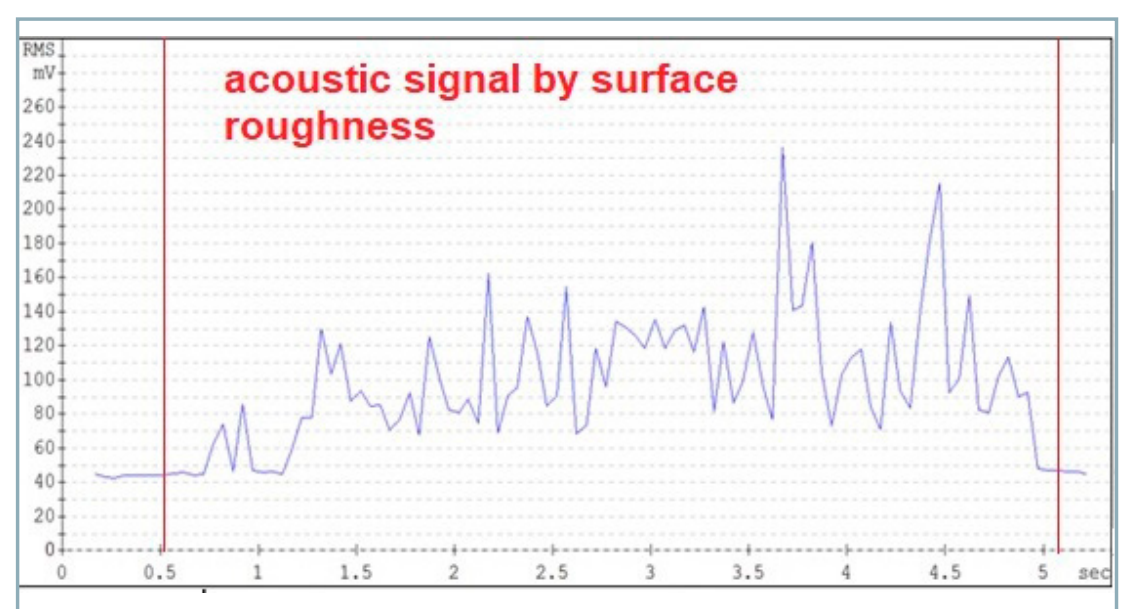

Fig. 5 Sample A3 measured using IDK-09 sensor on the $x$-axis

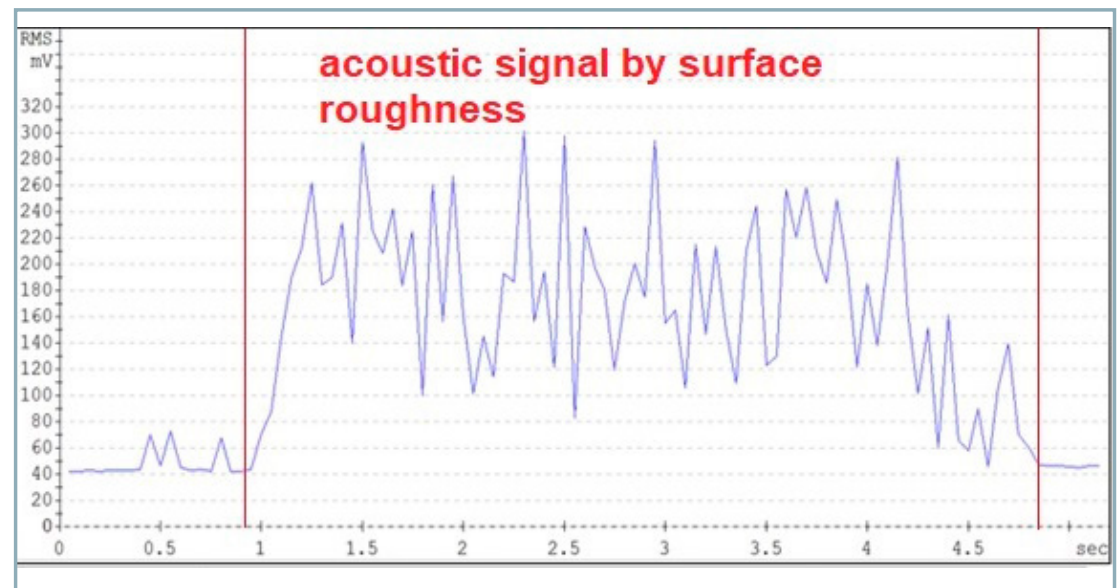

Fig. 6 Sample A3 measured using IDK-09 sensor on the $y$-axis

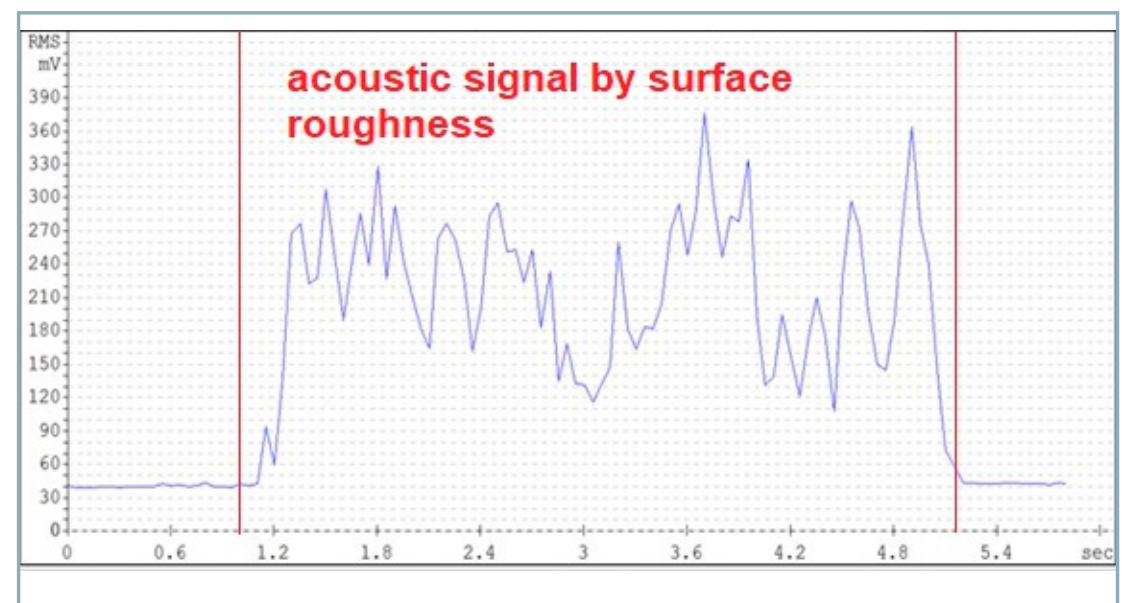

Fig. 7 Sample B5 measured using IDK-09 sensor on the $x$-axis diamond tip did not copy the surface, indicating that it is an inappropriate method for cast iron roughness measurements. The issue can be probably solved by using a diamond tip with a larger angle.

The last observed material was abrasion-resistant steel EN X53CrMnNiN21 9. The emission of acoustic waves is noticeable in both transverse and longitudinal directions with similar RMS values. It showed a suitable signal in regard to the measured voltage level on the sensor, as well as the shape, in both directions. This might have been caused by the surface finish of the material, which was performed by diamond grinding. This signal can be further processed and evaluated.

It is thus evident from the experimental results that hard materials with less rough surface are the most suitable materials for surface analysis using the AE method. Considering the softer materials and alloys, it is advisable to adjust the measuring diamond tip to avoid damaging the sample and prevent the influences on the measurement at the same time.

For the purposes of the experiment presented, a diamond cone with an angle of $60^{\circ}$ was selected to measure the surface roughness. Utilization of this apex angle on hard materials is considered appropriate, since the tip traces the surface more precisely and provides a more accurate picture of the surface. However, for surfaces with lower hardness, a diamond tip with a greater apex angle of $90^{\circ}$ would be more efficient. A larger apex angle should prevent the formation of a deep trace on the material surface and distorting the measurement results. In order to achieve the accurate results, gauge tip is made of diamond, because it shows high durability and also ensures low abrasion wear on surfaces with higher hardness. In the case of measuring the materials with lower hardness, it is also possible to use a cheaper alternative in the form of hardened steel.

The surface roughness $\mathrm{Ra}$ of specimens ranged from 1.6 to $3.2 \mu \mathrm{m}$. However, the voltage generated on the membrane differed considerably in observed materials. These deviations were caused by the hardness and 


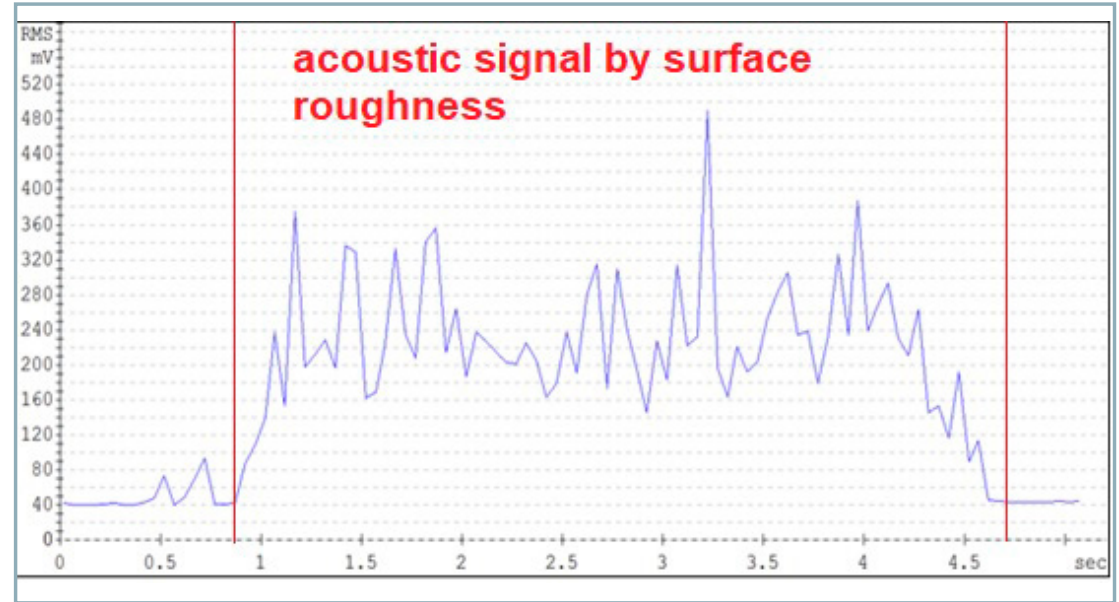

Fig. 8 Sample B5 measured using IDK-09 sensor on the $y$-axis

structure of the material. For example, the difference of the values in the particular axes is best seen in the $A 3$ sample (spheroidal graphite cast iron) where the voltage is at $130 \mathrm{mV}$ on the $y$-axis and at approx. $230 \mathrm{mV}$ on the $x$-axis.

\section{Conclusion}

The aim of this work was to investigate the suitability of the acoustic emission method for surface roughness measurement. The acoustic surface response of selected materials was observed. Diamond cone with apex angle $60^{\circ}$ was selected for the surface roughness measurement; utilization of this angle on hard materials is considered appropriate, because the tip follows the surface more accurately and provides a more accurate surface description. For surfaces with lower hardness, usage of a diamond tip with apex angle of at least $90^{\circ}$ would be more effective. A larger apex angle should prevent the formation of deep traces on the material surface and distorting the measurement results. For accurate results, the tip of the gauge uses a diamond that exhibits high durability and also ensures low wear on surfaces with higher hardness. In the case of materials with lower hardness, it is also possible to use a cheaper alternative in the form of hardened steel.

Surface roughness Ra ranged from 1.6 to $3.2 \mu \mathrm{m}$. However, the stress generated on the diaphragm varied widely between materials. These variations were a result of differences in the hardness and structure of materials, e.g. the difference in values in specific axes can be best seen in sample A3 (spheroidal graphite cast iron), in which the $y$-axis showed a voltage of $130 \mathrm{mV}$ and the $x$-axis approx. $230 \mathrm{mV}$.

This method is less suitable for hard materials with a surface roughness reaching $R a=3.2 \mu \mathrm{m}$. Furthermore, the measurements presented were greatly influenced by the directionality of the surface structure.

\section{Acknowledgements}

The research has been supported by the project IP 018/2019: Use of inorganic corrosion coatings for heterogeneous weldments protection; financed by IGA FA MENDELU.

\section{References}

BARÉNYI, I. - MAJERÍK, J. - POKORNÝ, Z. SEDLÁK, J. - BEZECNÝ, J. -

DOBROCKÝ, D. - JAROŠ, A. - ECKERT, M. JAMBOR, J. - KUSENDA, R. 2019 Material and technological investigation of machined surfaces of the OCHN3MFA steel. In Kovové Materiály, vol. 57, no. 2, pp. 131-142.

BHUSHAN, B. 2001. Chapter 2. Surface Roughness Analysis and Measurement Techniques, In BHUSHAN, B. Modern Tribology Handbook. New York : CRC Press, pp. 77. ISBN 0849384036.

BHUSHAN, B. 2013. Principles and Application of Tribology. John Wiley \& Sons, Ltd., 1006 pp. ISBN 978-1119944546.

DIAS, T. - PAULO, N. - QUEIJO, L. - LOPES, H. R. - CÉSAR, M. B. - RIBEIRO, J. E. 2016. Milling parameters optimization for surface quality. In CONTROLO 2016. Portuguese Conference in Automatic Control. Springer International Publishing Switzerland 2017, pp. 583-592. ISBN 9783319436715.
DOBROCKÝ, D. - KUSMIČ, D. 2015. The influence of the nitride layer depth in the root of V-notch to notch toughness of 30CRMOV9 steel. In Solid State Phenomena, vol. 258, pp. 242-545.

GONG, Y. - XU, J. - BUCHANAN, R. C. 2018. Surface roughness: $A$ review of its measurement at micro-/nano-scale. In Physical Sciences Reviews, vol. 3, no. 1, pp. 1-10.

JUNKI, J. - THOULESS, M. D. - BARBER, J. R. 2018. Effect of roughness on the adhesive tractions between contacting bodies. In Journal of the Mechanics and Physics of Solids, vol. 118, pp. 365-373.

OHTSU, M. 1995. The history and development of acoustic emission in concrete engineering. In Concrete Library JSCE, no. 25, pp. 121-134.

MACHEK, V. 2013. Metal Materials 1: Structure of Metal Materials. 1sted. Praha : České vysoké učení technické, 168 pp. ISBN 9788001052488. (In Czech: Kovové materiály 1: Struktury kovových materiálů). MILLER, R. - HILL, E. - MOORE, P. 2005. Nondestructive Testing Handbook: Acoustic Emission Testing:5. Columbus : American Society for Nondestructive, 603 pp. ISBN 9780931403026.

POLÁKOVÁ, N. - DOSTÁL, P. 2019. Titanium and stainless steel MIG LSC welding. In Acta Technologica Agriculturae, vol. 22, no. 2, pp. 56-59.

TULKA, J. 2005. Surface Treatment of Materials. $1^{\text {st }}$ ed. Brno : VUT, Fakulta chemická, 136 pp. ISBN 8021430621. (In Czech: Povrchové úpravy materiálů).

VOTAVA, J. - KUMBÁR, V. - POLCAR, A. - FAJMAN, M. 2020. Change of mechanical properties of zinc coating after heat treatment. In Acta Technologica Agriculturae, vol. 23, no. 1, pp. 7-11. 